J. Klin. Endokrinol. Stoffw. 2021 - 14:98-105 https://doi.org/10.1007/s41969-021-00146-9 Angenommen: 18. August 2021

๑ Der/die Autor(en) 2021

\section{Einleitung}

Der kongenitale Hyperinsulinismus (KHI) ist die häufigste Ursache persistierender Hypoglykämien im Säuglingsund Kindesalter; darunter präsentiert sich eine heterogene Gruppe von klinisch, genetisch und histologisch unterschiedlichen Erkrankungen [1, 2]. Charakterisiert ist der KHI durch eine überschießende Ausschüttung von Insulin und das Auftreten von Hypoglykämien, ohne Anstieg von Ketonkörpern oder freien Fettsäuren, die unbehandelt zu irreversiblen schweren neurokognitiven Defiziten führen.

Hyperinsulinämische Hypoglykämien können angeboren sein (Ursache: unterschiedliche Defekte im Pathway der Insulinsekretion der $\beta$-Zellen), mit verschiedenen Syndromen (z. B. BeckwithWiedemann-Syndrom, Sotos-Syndrom, Kabuki-Syndrom u.a.) assoziiert sein und auch auf metabolischen Ursachen, wie einem Defekt der Glykosylierung, wie z.B. im Rahmen des „congenital disorders of glycosylation (CDG) syndrome“, beruhen $[1,3,4]$. Zu den sekundären Ursachen zählen: maternaler Diabetes mellitus, Intrauterine growth restriction (IUGR) und perinatale Asphyxie $[1,4,5]$.

In früheren Jahren wurden Namen wie idiopathische Hypoglykämie, Nesidioblastose oder "persistent hyperinsulinemic hypoglycemia of infancy (PHHI)“ verwendet. In den letzten Jahren hat sich zunehmend der Begriff KHI als Überbegriff für eine Gruppe von verschiedenen genetischen Defekten durchgesetzt.

\title{
Birgit Rami-Merhar
}

Univ.-Klinik für Kinder- und Jugendheilkunde, Medizinische Universität Wien, Wien, Österreich

\section{Kongenitaler Hyperinsulinismus}

\section{Definition KHI}

Der KHI ist der Überbegriff für eine Erkrankungsgruppe, der folgende Kennzeichen gemeinsam sind:

- gestörte Regulation der Insulinsekretion,

- zu hohe Konzentration von Insulin, bezogen auf die Glucosekonzentration,

- rezidivierende Hypoglykämien und Erkrankung, die angeboren ist. Verschiedene genetische Defekte mit unterschiedlichem Vererbungsmuster können ursächlich zugrunde liegen.

Die Störung der Insulinsekretion kann so weitreichend sein, dass die Ausschüttung von Insulin fast vollständig von der Glucosekonzentration entkoppelt ist. Bei anderen Formen wird ständig zu viel Insulin ausgeschüttet; eine Abhängigkeit der Insulinsekretion von der Glucosekonzentration bleibt dabei aber erhalten $[1,2]$.

\section{Epidemiologie}

Die meisten Fälle von Hyperinsulinismus treten sporadisch auf. Für Mitteleuropa wird die Inzidenz des persistierenden KHI auf etwa 1:40.000 geschätzt. Bei einer Geburtenrate von rund 82.000 Kindern/Jahr in Österreich würde das 2 Fälle/Jahr betreffen, die Zahl liegt aber sicherlich etwas höher, da alleine die Medizinische Universität Wien 2 bis 4 Fälle/ Jahr betreut. Es gibt in Österreich aber kein entsprechendes Register. Das Auftreten familiärer Fälle variiert allerdings in verschiedenen ethnischen Regionen. Die Inzidenz kann in Abhängigkeit vom Grad an Konsanguinität in einer Bevölkerung bis auf 1:2500 ansteigen [1, 4].

\section{Unterschiedliche Formen des $\mathrm{KHI}$}

\section{Transienter neonataler Hyperinsulinismus}

Hypoglykämien bei gesunden Neugeborenen sind in den ersten Lebenstagen häufig und normalerweise auch selbstlimitierend [6, 7]. Ein transienter, neonataler Hyperinsulinismus kann bei folgenden Risikofaktoren auftreten: Frühgeburtlichkeit, IUGR, „large for gestational age" (LGA), perinatalem Stress (Rhesusinkompatibilität, perinataler Asphyxie, Mekoniumaspiration etc.). Mütterliche Risikofaktoren sind: Diabetes mellitus (v.a. Typ-1-Diabetes oder Gestationsdiabetes) oder eine antidiabetische Medikation (z. B. Sulfonylharnstoffe) $[1,2]$.

Die vorübergehende Hypoglykämieneigung kann bis zu 3 Monate nach der Geburt andauern, und typischerweise zeigt sich ein gutes Ansprechen auf Diazoxid. Ist der Kohlenhydratbedarf zur Aufrechterhaltung der Normoglykämie innerhalb der ersten Lebenswochen nicht eindeutig rückläufig, empfiehlt sich daher versuchsweise zunächst eine niedrig dosierte Diazoxidtherapie $[1,6,7]$.

\section{Insulinome im Kindesalter}

Insulinome sind im Kindesalter sehr selten, müssen aber differenzialdiagnostisch

\begin{tabular}{|ll}
\hline \multicolumn{2}{|l}{ Abkürzungen } \\
\hline CGMS & $\begin{array}{l}\text { "Continuous glucose monitoring } \\
\text { system" }\end{array}$ \\
\hline IUGR & "Intrauterine growth restriction" \\
\hline$K H I$ & Kongenitaler Hyperinsulinismus \\
\hline
\end{tabular}




\begin{tabular}{|c|c|c|c|c|c|}
\hline $\begin{array}{l}\text { Monogenetischer kongeni- } \\
\text { taler Hyperinsulinismus }\end{array}$ & Gen & Locus & Protein & Vererbung & $\begin{array}{l}\text { Ansprechen } \\
\text { auf Diazoxid }\end{array}$ \\
\hline \multicolumn{6}{|l|}{ KATP-Kanal } \\
\hline \multirow[t]{4}{*}{ Diffus } & \multirow[t]{2}{*}{$A B C C 8$} & \multirow[t]{2}{*}{$11 \mathrm{p} 15.1$} & \multirow[t]{2}{*}{ SUR1 } & $A R$ & $(-)$ \\
\hline & & & & $A D$ & $(-) /(+)$ \\
\hline & \multirow[t]{2}{*}{ KCNJ11 } & \multirow[t]{2}{*}{$11 \mathrm{p} 15.1$} & \multirow[t]{2}{*}{ Kir6.2 } & AR & $(-)$ \\
\hline & & & & $A D$ & $(+)$ \\
\hline \multirow[t]{2}{*}{ Fokal } & $A B C C 8$ & \multirow[t]{2}{*}{$11 \mathrm{p} 15.1$} & SUR1 & Sporadisch & $(-)$ \\
\hline & KCNJ11 & & Kir6.2 & $\begin{array}{l}\text { Väterliche } 11 \mathrm{p} \text {-UPD } \\
\text { und väterliche rezessive } \\
A B C C 8 / K C N J 11 \text {-Mutation }\end{array}$ & N.u. \\
\hline $\mathrm{GDH}$ & GLUD1 & $10 q 23.3$ & Glutamat-Dehydrogenase & Sporadisch/AD & $(+)$ \\
\hline GCK & GCK & $7 p 15$ & Glukokinase & Sporadisch/AD & $(+) /(-)$ \\
\hline $\mathrm{HADH}$ & $\mathrm{HADH}$ & $4 q 22-q 26$ & 3-Hydroxyacyl-Coenzym-A-Dehydrogenase & $A R$ & $(+)$ \\
\hline Pyruvattransporter (MCT-1) & SLC16A1 & $1 \mathrm{p} 13.2$ & Monocarboxylattransporter 1 & $A D$ & $(-) /(+)$ \\
\hline HNF4a & HNF4a & $20 q 13.12$ & „Hepatocyte nuclear factor $4 a^{\prime \prime}$ & $A D$ & $(+)$ \\
\hline HNF1a & HNF1a & $12 q 24.31$ & „Hepatocyte nuclear factor $1 a^{\prime \prime}$ & $A D$ & $(+)$ \\
\hline UCP2 & UCP2 & $11 q 13.4$ & „Uncoupling protein 2" & $A D$ & $(+)$ \\
\hline HK 1 & $H K 1$ & $10 q 21-22$ & Hexokinase 1 & $A D$ & $(+)$ \\
\hline PGM 1 & PGM 1 & $1 p .31 .3$ & Phosphoglukomutase 1 & $A R$ & $(-)$ \\
\hline PMM 2 & PMM 2 & 16p.13.2 & Phosphomannomutase 2 & $A R$ & $(+)$ \\
\hline CACNA1D & CACNA1D & $3 p 21.1$ & $\begin{array}{l}\text { "Calcium voltage-gated channel subunit a1 D“ } \\
\text { (spannungsabhängiger Kalziumkanal, Unter- } \\
\text { einheit a1 D) }\end{array}$ & Sporadisch & $(+)$ \\
\hline FOXA2 & FOXA2 & $20 p 11.21$ & „Forkhead box A2" & Sporadisch & $(+)$ \\
\hline
\end{tabular}

einbezogen werden. Sie treten meist erst deutlich später auf (> 10 Jahre) und manifestieren sich häufig mit einem Krampfanfall. Bei einem Insulinom im Rahmen einer multiplen endokrinen Neoplasie (MEN-1) muss ebenso nach Kennzeichen einer Neoplasie der Nebenschilddrüse und der Hypophyse gesucht werden. Die Familienanamnese kann hier ebenfalls weiterhelfen, MEN-1-Familien $\mathrm{zu}$ identifizieren [8].

\section{Monogenetische Formen des KHI}

Der KHI ist eine heterogene Erkrankung, mit persistierenden oder wiederkehrenden Hypoglykämien bei Hyperinsulinämie, ohne Anstieg von Ketonkörpern oder freien Fettsäuren. In den letzten Jahrzehnten konnten viele unterschiedliche Mutationen erforscht werden [1, 3, 9]. Sowohl die Vererbung als auch die Klinik können sehr unterschiedlich sein, eine rasche genetische Diagnostik ist aber für das weitere Management dieser Kinder essenziell und kann einen Hinweis darauf geben, ob es sich um eine diffuse oder fokale Form des KHI handelt (•Tab. 1; • Abb. 1).

Physiologisch führt ein Anstieg der intrazellulären Glucosekonzentration und des Metabolismus der Glucose unter Beteiligung des geschwindigkeitsbestimmenden Enzyms Glukokinase zu einem erhöhten ATP-ADP-Quotienten. Dies wird vom ATP-sensitiven Kaliumkanal $\left(\mathrm{K}_{\mathrm{ATP}}\right)$ registriert, der infolgedessen geschlossen wird. Daraus resultiert eine Änderung des Membranpotenzials mit Depolarisationen der Zellmembran, die wiederum $\mathrm{zu}$ einem Öffnen der spannungsabhängigen Kalziumkanäle führt. Der Kalziumeinstrom triggert die Exozytose von Insulin.

Pathophysiologisch können folgende Formen unterschieden werden:

- KATP-HI: Der ATP-sensitive Kaliumkanal ist defekt. Es kommt unabhängig von der Glucosekonzentration zu spontanen Depolarisationen und zu einer Exozytose von Insulin.

- GDH-HI: Überaktivität der Glutamatdehydrogenase (GDH).

Durch vermehrte Oxidation von Glutamat steigt der ATP/ADPQuotient. Dies steigert die Insulinsekretion.

- GCK-HI: Überaktivität der Glukokinase (GCK) führt zu einem, bezogen auf die Glucosekonzentration, erhöhten ATP/ADP-Quotienten und einer pathologisch erhöhten Insulinsekretion.

Klinisch überschneiden sich diese Formen allerdings, anhand des klinischen Bildes oder der Anamnese lassen sich keine sicheren Hinweise auf die mögliche genetische Ursache finden, daher ist eine rasche genetische Diagnostik wichtig. 


\section{KATP-Hyperinsulinismus: fokale und diffuse Form}

Die häufigste Form wird durch Defekte des ATP-sensitiven Kaliumkanals der $\beta$-Zelle verursacht, dieser besteht aus 2 Untereinheiten. Krankheitsauslösende Mutationen werden sowohl für das Gen des Sulfonylharnstoffrezeptors SUR1 (ABCC8) als auch für das Gen des Ionenkanals Kir6.2 (KCNJ11) beschrieben. Der Kaliumkanal ist entscheidend an der Regulation der Insulinsekretion beteiligt. Defekte in den kodierenden Genen des $\mathrm{K}_{\mathrm{ATP}}$ sind zumeist autosomal-rezessiv vererbt. Es wurden allerdings auch autosomal-dominant vererbte Mutationen beschrieben $[3,9]$.

\section{Fokale Form}

Lonlay et al. konnten 1997 molekulargenetisch nachweisen, dass es neben der diffusen Form des KATP-HI,verursacht durch Keimbahnmutationen, tatsächlich auch eine Form mit fokalen pankreatischen Veränderungen gibt [10]. Zwei gleichzeitig vorliegende Veränderungen in den $\beta$-Zellen der betroffenen Region führen zu einem fokalen KHI. Zum einen kommt es im Rahmen der Pankreasentwicklung in der fokalen Region zu einem Verlust der Heterozygotie für die Region 11 p15 durch den Verlust des maternalen Allels. Dadurch liegt eine paternal vererbte Keimbahnmutation (ABCC8-, KCNJ11-Gen) in dieser Region homozygot oder hemizygot vor, da das normale maternale Allel fehlt, und führt in den betroffenen $\beta$-Zellen zum Hyperinsulinismus. Zum anderen handelt es sich bei der Region 11p15 um eine „imprinted region", in der die Gene in Abhängigkeit von paternaler oder maternaler Vererbung exprimiert werden [11]. Der Verlust von u.a. maternal exprimierten Tumorsuppressorgenen führt $\mathrm{zu}$ einem klonalen Wachstum der betroffenen Zellen und resultiert in der adenomatösen Veränderung. Die Häufigkeit des fokalen Hyperinsulinismus wird mit bis $\mathrm{zu}$ $40 \%$ bei pankreatektomierten Patienten angegeben.

Es gibt weitere Formen des KHI, wie den Glukokinase-Hyperinsulinismus (autosomal-dominant, aktivierende Mutation mit gesteigerter Glucoseutilisation), den Glutamatdehydrogenase-
Hyperinsulinismus (autosomal-dominant, aktivierende Mutation im GLUD1Gen der mitochondrialen Glutamatdehydrogenase $(\mathrm{GDH})$ ) und den SCHADHyperinsulinismus („Short-chain-L-3Hydroxylacyl-coenzyme-A“-Dehydrogenasemangel). Weitere, noch seltenere Formen sind ebenfalls beschrieben [1, 7].

Ein Hyperinsulinismus, der zu Hypoglykämien führt, kann auch das erste Symptom verschiedener syndromaler Erkrankungen sein (z. B. Beckwith-Wiedemann-Syndrom, Sotos-Syndrom, Kabuki-Syndrom u.a.).

\section{Klinik}

Ein Verdacht auf einen KHI kann bereits pränatal gestellt werden, wenn eine Makrosomie festgestellt wurde, diese ist bedingt durch die Hyperinsulinämie. Die meisten Säuglinge mit neonataler Manifestation fallen innerhalb der ersten 24 Lebensstunden durch hypoglykämietypische Symptome (u.a. Krampfanfälle, Zyanose, Apathie, Apnoen, Schwitzen, Zittern) auf. Bei einer späteren Manifestation im Säuglings- oder Kleinkindalter tritt sehr oft ein Krampfanfall im Rahmen der Hypoglykämien und damit einhergehenden Minderversorgung des Gehirns mit Glucose auf. Mildere Symptome wie Schwäche, Schwindel, Müdigkeit, Sprachstörungen oder Verwirrtheit können ebenso Hinweise auf Hypoglykämien sein.

Der weitere klinische Verlauf ist abhängig von einer frühzeitigen Diagnosestellung und Einleitung einer suffizienten Therapie zur Vermeidung einer zerebralen Schädigung durch wiederholte Hypoglykämien.

Bei Neugeborenen ist die Berechnung des Kohlenhydratbedarfs zur Aufrechterhaltung der Normoglykämie ein wichtiges diagnostisches Kriterium. Ein Bedarf $>10 \mathrm{mg} / \mathrm{kgKG}$ pro min spricht für einen Hyperinsulinismus. Für die sichere Glucosezufuhr ist hier meist ein zentraler Zugang notwendig, um die hochprozentigen Glucoselösungen verabreichen $\mathrm{zu}$ können.

Kontinuierliche Glucosemesssysteme (CGMS) sind eigentlich nicht für das Säuglingsalter zugelassen. Die Messge-
J. Klin. Endokrinol. Stoffw. $2021 \cdot 14$ 98-105

https://doi.org/10.1007/s41969-021-00146-9 (c) Der/die Autor(en) 2021

\section{B. Rami-Merhar \\ Kongenitaler Hyperinsulinismus}

\section{Zusammenfassung}

Der kongenitale Hyperinsulinismus (KHI) ist die häufigste Ursache persistierender Hypoglykämien im Säuglings- und Kindesalter; darunter präsentiert sich eine heterogene Gruppe von klinisch, genetisch und histologisch unterschiedlichen Erkrankungen. Charakterisiert ist der KHI durch eine überschießende Ausschüttung von Insulin und das Auftreten von Hypoglykämien, die unbehandelt zu schweren irreversiblen neurokognitiven Defiziten führen, daher ist eine rasche Diagnose und Behandlung essenziell. In den letzten Jahren gab es viele neue Erkenntnisse im Bereich der Genetik, Diagnostik und Therapie.

\section{Schlüsselwörter}

Hypoglykämie · Kongenitaler Hyperinsulinismus · Säuglinge · Kleinkinder · Diffuse und fokale Formen

\section{Congenital hyperinsulinism}

\section{Abstract}

Congenital hyperinsulinism $(\mathrm{CHI})$ is the most frequent cause of persisting hypoglycemia in infancy and childhood and includes a heterogeneous group of clinically, genetically and histologically different diseases. The $\mathrm{CH}$ is characterized by an overproduction of insulin and the occurrence of hypoglycemia, which leads to neurocognitive deficits if not treated immediately. Rapid diagnosis and treatment are essential to avoid irreversible hypoglycemic brain damage. In recent years a great deal of new knowledge has been gained in the fields of genetics, diagnostics and treatment.

\section{Keywords}

Hypoglycemia - Congenital hyperinsulinism . Babies · Infants · Diffuse and focal forms

nauigkeit der CGMS-Systeme ist aber ähnlich wie bei größeren Kindern und wurden daher auch schon erfolgreich bei Säuglingen mit KHI verwendet [12]. Darunter kann die Messfrequenz der kapillären Blutzuckermessungen (teilweise stündlich notwendig) reduziert werden. 


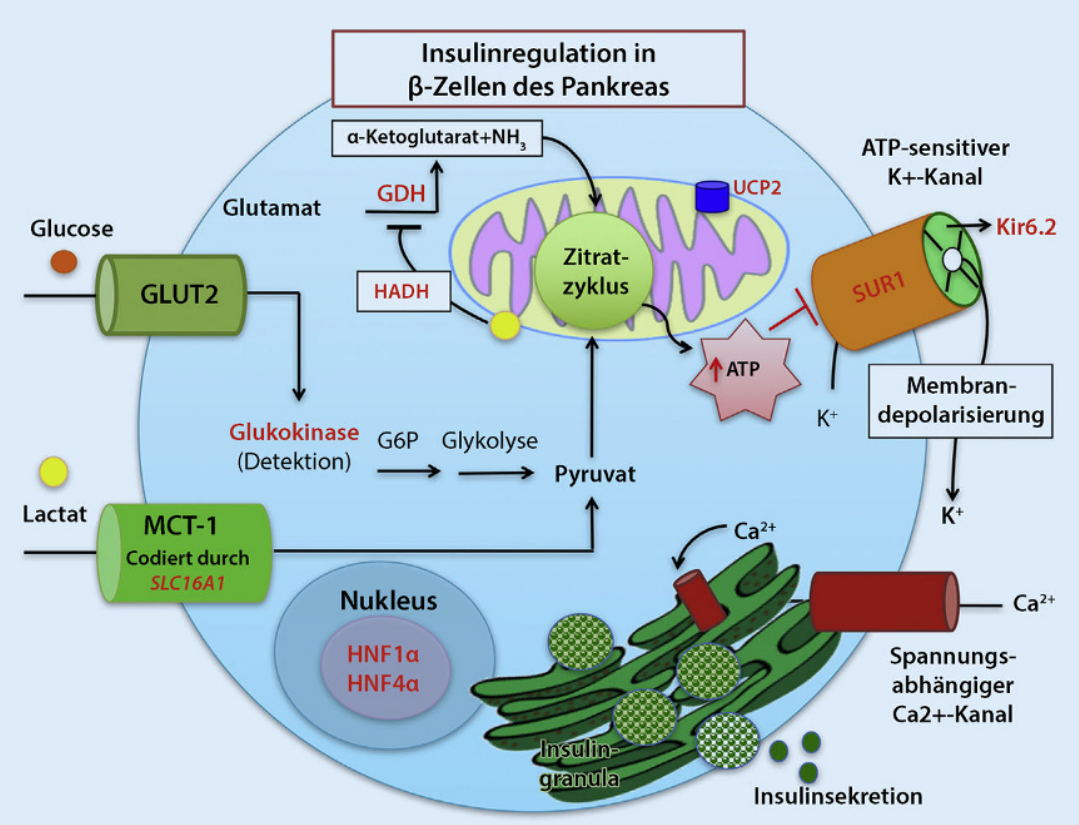

Abb. 1 A Darstellung der Regulation der Insulinsekretion sowie Pathomechanismen der wichtigsten Formen des KHI [1]. ATP Adenosintriphosphat, $\mathrm{Ca}^{2+}$ Kalziumionen, G6P Glukose-6-Phosphat, GDH Glutamatdehydrogenase, GLUT2 Glucosetransporter 2, HADH 3-Hydroxyacyl-Koenzym-A-Dehydrogenase, HNF1a ", hepatocyte nuclear factor $1 a^{\prime \prime}, H N F 4 a$ ", hepatocyte nuclear factor $4 a^{\prime \prime}, K^{+}$Kalium, Kir6.2 „inward rectifier potassium channel 6.2“ (Kaliumkanal), MCT-1 Monocarboxylattransporter 1 , $\mathrm{NH}_{3}$ Ammoniak, SLC16A1 "solute carrier family 16, member 1", SUR1 "sulfonylurea receptor 1" (Sulfonylharnstoffrezeptor 1), UCP2 "mitochondrial uncoupling protein 2". (Aus Galcheva et al. [1], mit freundlicher Genehmigung von Elsevier. Copyright $\odot 2018$. Elsevier Inc. All rights reserved. Diese Abbildung fällt nicht unter die Creative Commons CC BY-Lizenz dieser Publikation.)

Typischerweise lässt sich bei KHI im Rahmen der Hypoglykämie $(<45 \mathrm{mg} / \mathrm{dl})$ ein erhöhter Insulinspiegel $(>3 \mathrm{mU} / \mathrm{l})$ finden und ergibt damit den ersten Hinweis auf die Pathophysiologie. Typisch ist der Nachweis eines messbaren Insulinspiegels im Rahmen der Hypoglykämie, ohne Anstieg von Ketonkörpern oder freien Fettsäuren. Zumeist finden sich bei anderen Ursachen von Hypoglykämien zum Zeitpunkt der Unterzuckerung keine messbaren Insulinkonzentrationen. Die von Laboren vorgegebenen Referenzwerte für die Nüchterninsulinkonzentration (von z. B. 3-17 mU/l) können missverständlich sein, da die bei KHI gemessenen Werte oft in diesem Normalbereich liegen (selten $>100 \mathrm{mU} / \mathrm{l}$ ) [13].

Die Gabe von Glukagon hat einen Stellenwert, um das Ansprechen auf das Medikament in der Behandlung von Hypoglykämien abschätzen zu können; weniger geeignet ist die Gabe von Glukagon als diagnostisches Hilfsmittel, da fast alle Formen der Hypoglykämie (außer Glykogenosen) darauf ansprechen.

Bei klinisch leichteren Formen ist die Diagnose oft schwieriger zu stellen, insbesondere wenn der Bedarf an Glucose zur Aufrechterhaltung der Normoglykämie nicht erhöht ist. Hier können wiederholte Messungen der Insulinkonzentration sowie die sorgfältige Durchführung eines Fastentestes (nach Ausschluss einer Fettsäurenoxidationsstörung) weiterhelfen. Eine wichtige Differenzialdiagnose ist bei Neugeborenen neben den transienten Hypoglykämien ein Panhypopituitarismus wie z. B. bei einer septooptischen Dysplasie.

\section{Diagnosestellung}

Die Diagnosestellung bei den schweren Formen des KHI mit neonataler Mani-

\section{Hier steht eine Anzeige.}

\section{型 Springer}




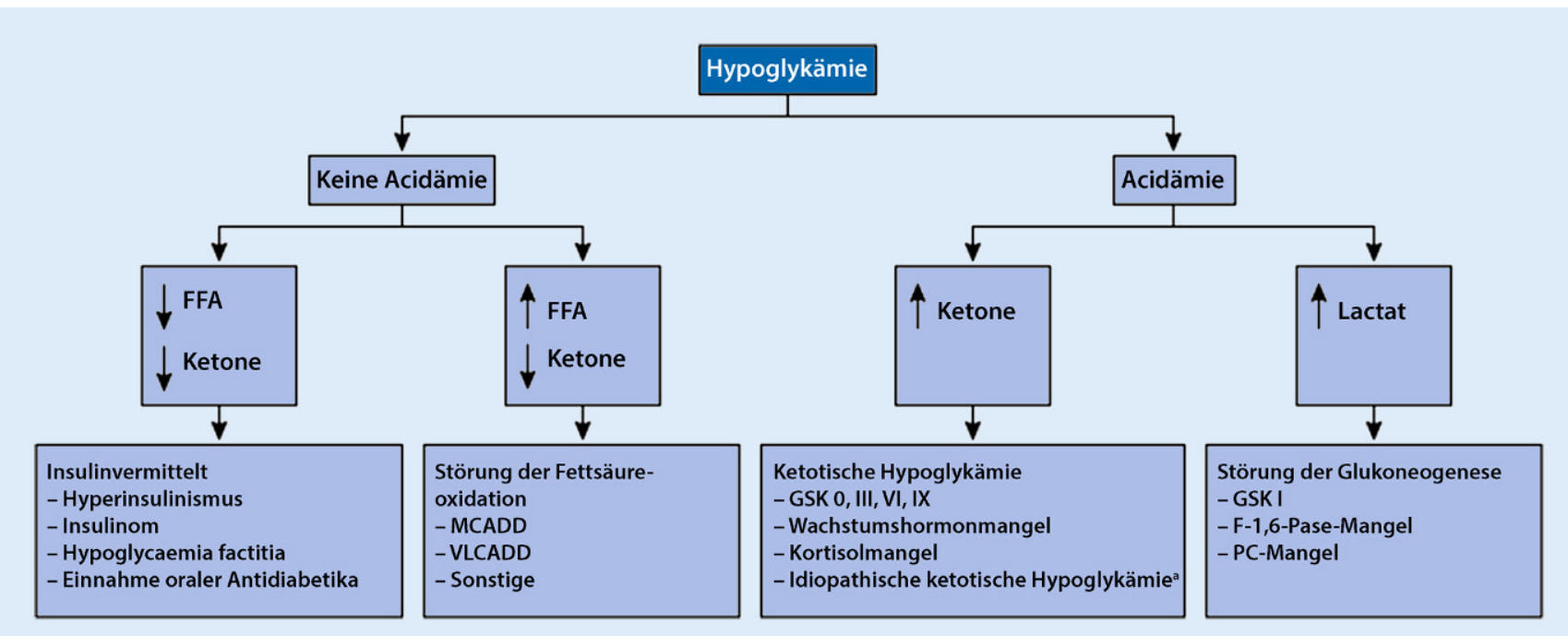

Abb. $2 \Delta$ Flowchart zur Kategorisierung der zugrunde liegenden Ursache der Hypoglykämie. FFA "free fatty acids" (freie Fett-

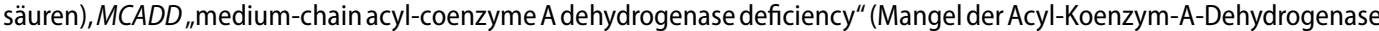
für mittelkettige Fettsäuren), VLCADD "very-long-chain acyl-coenzyme A dehydrogenase deficiency" (Mangel der Acyl-Koenzym-A-Dehydrogenase für sehr langkettige Fettsäuren), GSK Glykogenspeicherkrankheit, F-1,6-Pase Fruktose-1,6-Bisphosphatase, PC Pyruvatcarboxylase. a Bei manchen ketotisch-hypoglykämischen Störungen liegt möglicherweise keine messbare Acidämie vor, insbesondere bei idiopathischer ketotischer Hypoglykämie, Wachstumshormon- und Kortisolmangel. (Mod. nach Thornton et al. [7])
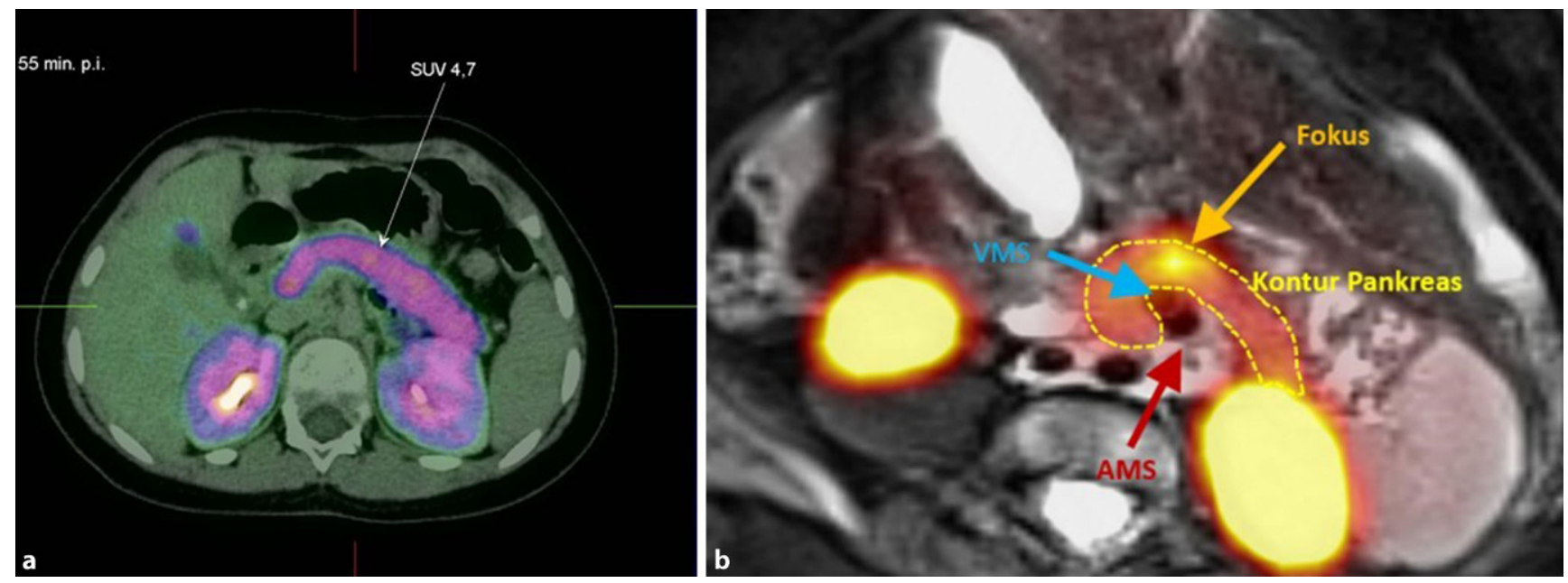

Abb. $3 \Delta$ a Darstellung einer diffusen Form eines Hyperinsulinismus mittels $\left[{ }^{18} \mathrm{~F}\right]$ Fluor-DOPA-PET-CT. b Darstellung einer fokalen Form mittels Fusion $\left.{ }^{[68} \mathrm{Ga}\right]-E x e n d i n-P E T$ und T2 SPACE. (Eigene Patienten, Bilder aus der Charité Berlin mit freundlicher Genehmigung. Diese Abbildung fällt nicht unter die Creative Commons CC BY-Lizenz dieser Publikation.)

festation gelingt im Normalfall rasch; die möglichen Differenzialdiagnosen der Hypoglykämie sind im Flowchart in - Abb. 2 dargestellt.

Nach der Sicherung der Diagnose und unter Sicherung einer Normoglykämie mittels Glucoseinfusion und Start einer medikamentösen Therapie ist der nächste Schritt eine rasche genetische Diagnostik, um idealerweise diffuse von fokalen Formen eines KHI unterscheiden zu können. Bei der diffusen Form sind alle $\beta$-Zellen betroffen. Die fokale Form weist eine oder mehrere Regionen mit adenomatösen Veränderungen auf. Abhängig vom genetischen Befund sind weitere Untersuchungen zielführend, es muss aber erwähnt werden, dass derzeit in nur rund $50 \%$ der Fälle auch eine genetische Ursache gefunden werden kann.

\section{Bildgebung}

Bei genetisch gesicherten diffusen Formen des KHI ist keine weitere Bildgebung indiziert, und die konservative Therapie muss so adaptiert werden, dass die Glucoseinfusion möglichst beendet werden kann. Bei genetisch gesicherten fokalen Formen oder unklarer Genetik (rund $50 \%$ der Fälle) ist als nächster Schritt ein [18F]Fluor-DOPA-PET-CT zur Dif- 
Tab. 2 Auflistung derverfügbaren Medikamente und Dosierungen. Diazoxid, Octreotid und Lanreotid sind die Medikamente, die in den letzten Jahren den größten Therapierfolg gezeigt haben. (Aus Galcheva et al. [1], mit freundlicher Genehmigung von Elsevier. Copyright $\odot$ 2018. Elsevier Inc. All rights reserved. Diese Tabelle fällt nicht unter die Creative Commons CC BY-Lizenz dieser Publikation.)

\begin{tabular}{|c|c|c|c|c|}
\hline Wirkstoff & Applikationsweg & Dosis & Wirkmechanismus & Nebenwirkungen \\
\hline Diazoxid & Oral & $\begin{array}{l}5-20 \mathrm{mg} / \mathrm{kgKG} \text { und Tag, } \\
\text { verteilt auf } 3 \text { Dosen }\end{array}$ & $\begin{array}{l}\text { KATP-Kanal-Agonist; für } \\
\text { die Wirkung ist eine } \\
\text { erhaltene KATP-Kanal- } \\
\text { Aktivität erforderlich }\end{array}$ & $\begin{array}{l}\text { Häufig: Wasser- und Natriumretention, Hypertrichose, } \\
\text { Appetitverlust. Selten: Herzinsuffizienz, Hyperurikämie, Hy- } \\
\text { potonie, Leukopenie, Eosinophilie, paradoxe Hypoglykämie, } \\
\text { pulmonale Hypertonie }\end{array}$ \\
\hline $\begin{array}{l}\text { Chlorothiazid } \\
\text { (in Kombi- } \\
\text { nation mit } \\
\text { Diazoxid) }\end{array}$ & Oral & $\begin{array}{l}7-10 \mathrm{mg} / \mathrm{kgKG} \text { und Tag, } \\
\text { verteilt auf } 2 \text { Dosen }\end{array}$ & $\begin{array}{l}\text { Verhindert Flüssig- } \\
\text { keitsretention; syner- } \\
\text { gistische Effekte mit } \\
\text { Diazoxid am KATP-Kanal }\end{array}$ & Hyponatriämie, Hypokaliämie \\
\hline Nifedipin & Oral & $\begin{array}{l}0,25-2,5 \mathrm{mg} / \mathrm{kgKG} \text { und } \\
\text { Tag in } 2 \text { bis } 3 \text { Dosen }\end{array}$ & Kalziumkanalblocker & Hypotonie \\
\hline Glukagon & $\begin{array}{l}\text { s.c./i.m.-Boli oder } \\
\text { kontinuierliche } \\
\text { s.c./i.v.-Infusion }\end{array}$ & $\begin{array}{l}0,5-1,0 \mathrm{mg} \text { als Bo- } \\
\text { lus oder Infusion von } \\
1-10 \mu \mathrm{g} / \mathrm{kgKG} \text { und } \mathrm{h}\end{array}$ & $\begin{array}{l}\text { Verstärkt Glykogenoly- } \\
\text { se oder Glukoneogene- } \\
\text { se }\end{array}$ & $\begin{array}{l}\text { Übelkeit, Erbrechen, Hautausschlag, paradoxe Insulinse- } \\
\text { kretion und Rebound-Hypoglykämie (nach Verwendung } \\
\text { höherer Dosen) }\end{array}$ \\
\hline Octreotid & $\begin{array}{l}\text { Alle } 6-8 \mathrm{~h} \text { als s.c.- } \\
\text { Injektion oder } \\
\text { kontinuierliche } \\
\text { s.c.-Infusion }\end{array}$ & $5-35 \mu \mathrm{g} / \mathrm{kgKG}$ und Tag & $\begin{array}{l}\text { Somatostatinanalogon, } \\
\text { hemmt Insulinsekreti- } \\
\text { on, reduziert Insulin- } \\
\text { biosynthese, wirkt an } \\
\text { KATP-Kanälen }\end{array}$ & $\begin{array}{l}\text { Akut: Anorexie, Übelkeit, aufgetriebenes Abdomen, medi- } \\
\text { kamenteninduzierte Hepatitis, Steatorrhö, Long-QT-Syn- } \\
\text { drom, Tachyphylaxie, nekrotisierende Enterokolitis } \\
\text { Langfristig: verminderte Darmmotilität, Gallenschlamm, } \\
\text { Cholelithiasis, Suppression von Wachstumshormon, thy- } \\
\text { reoidstimulierendem Hormon und adrenokortikotropem } \\
\text { Hormon }\end{array}$ \\
\hline $\begin{array}{l}\text { Lanreotid } \\
\text { oderlang } \\
\text { wirksames } \\
\text { Octreotid }\end{array}$ & i.m./tief s.c. & $\begin{array}{l}\text { Applikation der Ge- } \\
\text { samtdosis von Octreotid } \\
60 \mathrm{mg} \text { alle } 4 \text { Wochen }\end{array}$ & $\begin{array}{l}\text { Lang wirksame Soma- } \\
\text { tostatinanaloga mit } \\
\text { ähnlichen Wirkungen } \\
\text { wie die mehrfach täg- } \\
\text { liche Applikation von } \\
\text { Octreotid }\end{array}$ & $\begin{array}{l}\text { Ähnlich wie bei mehrfach täglicher Octreotidinjektion; } \\
\text { allerdings liegen keine Langzeitdaten vor }\end{array}$ \\
\hline Sirolimus & Oral & $\begin{array}{l}\text { Therapiebeginn mit } \\
0,5 \mathrm{mg} / \mathrm{m}^{2} \mathrm{KOF} \text { und } \\
\text { Tag in } 1 \text { bis } 2 \text { Dosen; } \\
\text { Dosisanpassung zur } \\
\text { Aufrechterhaltung } \\
\text { therapeutischer Spiegel }\end{array}$ & $\begin{array}{l}\text { mTOR-Inhibitor, } \\
\text { hemmt } \beta \text {-Zell-Proli- } \\
\text { feration und Insulinse- } \\
\text { kretion, Mechanismen } \\
\text { nicht voll verstanden }\end{array}$ & $\begin{array}{l}\text { Stomatitis, erhöhtes Infektionsrisiko, Immunsuppression, } \\
\text { Abweichungen in der Nierenfunktion, Fatigue, Pneumonitis, } \\
\text { Hyperlipidämie, vorübergehend erhöhte Leberenzyme, } \\
\text { Thrombozytose, gestörte Immunreaktion auf BCG-Vakzine }\end{array}$ \\
\hline
\end{tabular}

ferenzierung von fokaler und diffuser Form des KHI durchzuführen. Weitere angewandte nuklearmedizinische Tracer sind DOTATOC oder Exendin-4, jeweils in Kombination mit einer hochauflösenden Bildgebung ([14-16]; • Abb. 3). Diese Untersuchungen sollten aber nach Möglichkeit in einem Zentrum durchgeführt werden, das viel Erfahrung mit dieser spezifischen Untersuchung bei jungen Kindern hat. Aufgrund der Seltenheit dieser Fragestellung werden die meisten österreichischen Kinder für diese Untersuchung nach Deutschland überwiesen.

\section{Therapie}

Nach der gesicherten Diagnose eines KHI und Substitution von Glucose (meist i.v. und teilweise mit einem Bedarf von
$>20 \mathrm{mg} / \mathrm{kg}$ und $\mathrm{min}$ ) wird eine konservative Therapie initialisiert.

Eine diätetische Therapie in Form von häufigen Mahlzeiten und kohlenhydratreicher Ernährung kann zur Stabilisierung der Glucosewerte beitragen. Bei Säuglingen können Reisflocken zur Muttermilch oder zur Milchnahrung hinzugefügt, bei Kindern nach dem 1 . bis 2. Lebensjahr kann auch ungekochte Maisstärke verwendet werden. Die verfügbaren Medikamente sind in •Tab. 2 aufgelistet.

\section{Konservative Therapie}

Diazoxid öffnet den $K_{\text {ATP }}$ der $\beta$-Zelle, daher spricht dieses Medikament bei einem $K_{\mathrm{ATP}}-\mathrm{HI}$, bei dem dieser $\mathrm{K}_{\mathrm{ATP}}$ Kanal defekt ist, meist nicht an und der Hyperinsulinismus besteht weiter- hin [17]. Von einem Ansprechen auf Diazoxid sollte nur im Fall einer Normalisierung des Kohlenhydratbedarfs gesprochen werden. Durch zusätzliche Gabe eines Thiazids (z. B. Hydrochlorothiazid) kann die Flüssigkeitsretention, die v. a. bei höheren Dosen von Diazoxid auftritt, reduziert werden. Unter prolongierter Diazoxidtherapie kommt es v.a. bei höherer Dosierung regelmäßig zu einer Hypertrichose (• Abb. 4).

Da spannungsabhängige Kalziumkanäle an der Regulation der Insulinsekretion beteiligt sind, wurden auch Kalziumantagonisten verwendet, hier sind die Erfahrungen aber limitiert, und die klinische Erfahrung hat wenig Erfolge gezeigt. Behandlungsversuche mit Sirolimus wurden nur in Einzelfällen bei sehr schweren Verlaufsformen des KHI eingesetzt, aufgrund der Nebenwir- 


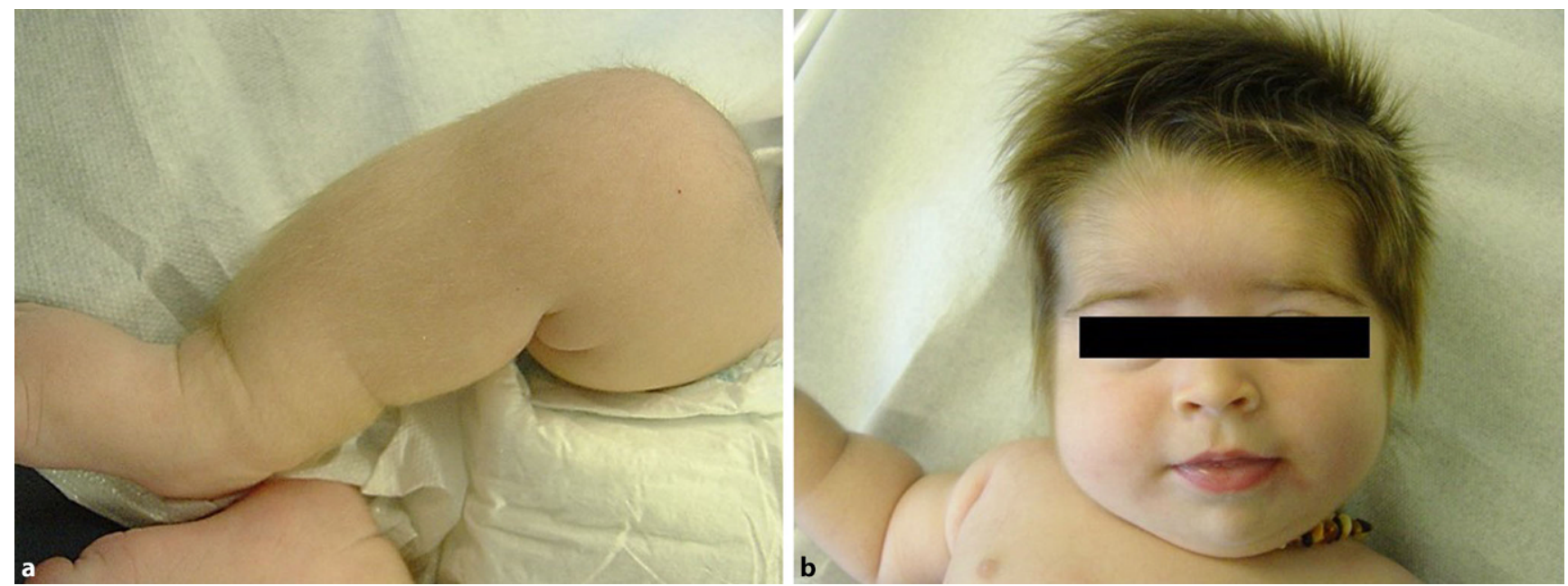

Abb. 4 A Hypertrichose unter einer Therapie mit Diazoxid. a Am Unterschenkel, b am Kopf. (Eigene Bilder, mit freundlicher Genehmigung der Familie)

kungen (Immunsuppressivum) wird es derzeit nicht empfohlen. Glukagon als i.v.-Gabe kann in Phasen von exzessivem Glucosebedarf verwendet werden, eignet sich aber nicht als Dauertherapie.

Wenn die Glucosezufuhr unter Diazoxid nicht reduziert werden kann, dann ist Octreotid das Medikament der Wahl. Dieses wird initial 3-mal täglich s.c. verabreicht; bei Ansprechen kann Octreotid auch als Dauerinfusion mittels Insulinpumpe angewendet werden. Bei länger dauernder Anwendung kann auf das lang wirksame Lanreotid (Gabe alle 4 bis 6 Wochen s.c.) umgestellt werden. Auch hier müssen mögliche Nebenwirkungen (• Tab. 2) gut monitiert werden.

Die Familien müssen bezüglich rascher Behandlung von Hypoglykämien mit oraler Gabe von Glucose oder in Notfällen Glukagon geschult werden. Die Verwendung von CGMS erleichtert den Alltag dieser Familien und die Integration in Kindergarten und Schule.

Weitere Medikamente sind in Erprobung, jedoch bisher nur im Rahmen von klinischer Forschung.

\section{Operative Therapie}

Beim transienten Hyperinsulinismus sind operative Eingriffe nicht indiziert. Fälle, bei denen in der speziellen Bildgebung (z.B. $\left[{ }^{18} \mathrm{~F}\right]$ Fluor-DOPA-PET-CT) der Verdacht auf eine fokale oder adenomatöse Form gestellt wird, und die einen schwereren Verlauf haben, sollten nach Abwägung aller Risiken operiert werden. Das intraoperative Auffinden der fokalen Läsionen ist oft schwierig, da sie makroskopisch oft nicht sichtbar sind. Die Operationen sollten nur in ausgewählten Zentren mit entsprechender Erfahrung und technischen Voraussetzungen (Histologie, intraoperative Bildgebung) durchgeführt werden.

Auch bei diffusen Formen, die sich medikamentös nicht zufriedenstellend behandeln lassen, kann eine partielle, manchmal sogar fast vollständige Pankreatektomie (>90\%) notwendig werden. Während ein geringeres Ausmaß der Pankreasresektion alleine oft unwirksam ist, kann die medikamentöse Therapie häufig nach einer Teilresektion effektiver werden. Nicht selten ist jedoch eine Nachresektion notwendig, wenn postoperativ die Hypoglykämien persistieren. Mit zunehmender Größe der Resektion steigt das Risiko einer exokrinen Pankreasinsuffizienz und eines Diabetes mellitus (Typ 3). Eine Operation sollte nur dann in Betracht gezogen werden, wenn alle konservativen Maßnahmen ausgeschöpft sind.

\section{Prognose}

Publikationen zu Langzeitbeobachtungen von Patienten mit KHI sind rar und meistens schon älter $[17,18]$. Die darin berichteten hohen Zahlen an Pankreatektomien und Komplikationen wie z.B. mentaler Retardierung entsprechen sicherlich nicht mehr dem aktuellen Standard. Das Risiko für eine psychomotorische Retardierung oder für die Entwicklung einer Epilepsie ist nicht allein von der Schwere des Hyperinsulinismus abhängig, sondern auch von einer frühzeitigen Diagnosestellung [19]. Durch die moderneren Verfahren (inkl. Bildgebung, Genetik, CGMS, Medikamente) konnte sicherlich eine Verbesserung erzielt werden; zumindest zeigt das die klinische Erfahrung. Essenziell sind auf jeden Fall eine rasche Diagnose und die Vermeidung von Hypoglykämien (oder deren rasche Behandlung), um eine normale Entwicklung zu erreichen.

Die klinische Erfahrung hat gezeigt, dass es Patienten gibt, die spontan in Remission kommen, aber noch ist nicht klar, welche Patienten das betrifft und wann diese zu erwarten ist. Öfter zeigt sich, dass die Dosis der Medikamente bei konservativ behandelten Patienten mit zunehmendem Alter reduziert oder auch beendet werden kann.

\section{Fazit für die Praxis}

Eine rasche Diagnostik und Therapie ist bei kongenitalem Hyperinsulinismus im Säuglings- und Kindesalter essenziell, um irreversible schwere neurokognitive Defizite verhindern zu können. 


\section{Korrespondenzadresse

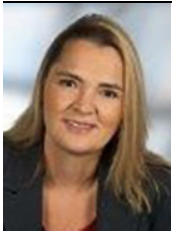 \\ Univ.-Prof. Dr. Birgit Rami-Merhar, MBA \\ Univ.-Klinik für Kinder- und Jugendheilkunde, Medizinische Universität Wien \\ Wien, Österreich \\ Birgit.Rami@ meduniwien.ac.at}

Funding. Open access funding provided by Medical University of Vienna.

\section{Einhaltung ethischer Richtlinien}

Interessenkonflikt. B. Rami-Merhar gibt an, dass kein Interessenkonflikt besteht.

Für diesen Beitrag wurden von der Autorin keine Studien an Menschen oder Tieren durchgeführt. Für die aufgeführten Studien gelten die jeweils dort angegebenen ethischen Richtlinien.

Open Access. Dieser Artikel wird unter der Creative Commons Namensnennung 4.0 International Lizenz veröffentlicht, welche die Nutzung, Vervielfältigung Bearbeitung, Verbreitung und Wiedergabe in jeglichem Medium und Format erlaubt, sofern Sie den/die ursprünglichen Autor(en) und die Quelle ordnungsge mäß nennen, einen Link zur Creative Commons Lizenz beifügen und angeben, ob Änderungen vorgenommen wurden.

Die in diesem Artikel enthaltenen Bilder und sonstiges Drittmaterial unterliegen ebenfalls der genannten Creative Commons Lizenz, sofern sich aus der Abbildungslegende nichts anderes ergibt. Sofern das betreffende Material nicht unter der genannten Creative Commons Lizenz steht und die betreffende Handlung nicht nach gesetzlichen Vorschriften erlaubt ist, ist für die oben aufgeführten Weiterverwendungen des Materials die Einwilligung des jeweiligen Rechteinhabers einzuholen.

Weitere Details zur Lizenz entnehmen Sie bitte der Lizenzinformation auf http://creativecommons.org/ licenses/by/4.0/deed.de.

\section{Literatur}

1. Galcheva S, Al-Khawaga S, Hussain K (2018) Diagnosis and management of hyperinsulinaemic hypoglycaemia. Best Pract Res Clin Endocrinol Metab 32:551-573

2. Shah P, Rahman SA, Demirbilek H, Güemes M, Hussain $\mathrm{K}$ (2017) Hyperinsulinaemic hypoglycaemia in children and adults. Lancet Diabetes Endocrinol 5:729-742

3. Kapoor RR et al (2013) Clinical and molecular characterisation of 300 patients with congenital hyperinsulinism. Eur JEndocrinol 168:557-564

4. Senniappan S, Arya VB, Hussain K (2013) The molecular mechanisms, diagnosis and management of congenital hyperinsulinism. Indian J Endocrinol Metab 17:19-30
5. Arya VB et al (2014) Clinical and histological heterogeneity of congenital hyperinsulinism due to paternally inherited heterozygous $A B C C 8 / K C N J 11$ mutations. Eur J Endocrinol 171:685-695

6. Stanley CA, Rozance PJ, Thornton PS, De Leon DD, Harris D, Haymond MW, Hussain K, Levitsky LL Murad MH, Simmons RA, Sperling MA, Weinstein DA, White NH, Wolfsdorf JI (2015) Reevaluating "transitional neonatal hypoglycemia": mechanism and implications for management. JPediatr 166:1520-1525

7. Thornton PS et al (2015) Recommendations from the Pediatric Endocrine Society for Evaluation and Management of Persistent Hypoglycemia in Neonates, Infants, and Children. J Pediatr 167:238-245

8. Shin JJ, Gorden P, Libutti SK (2010) Insulinoma: pathophysiology, localization and management. Future Oncol 6:229-237

9. De Franco $E$ et al (2020) Update of variants identified in the pancreatic $\beta$-cell KATP channe genes $\mathrm{KCNJ} 11$ and $A B C C 8$ in individuals with congenital hyperinsulinism and diabetes. Hum Mutat 41:884-905

10. de Lonlay P, Fournet JC, Rahier J, Gross-Morand MS, Poggi-Travert F, Foussier V, Bonnefont JP, Brusset MC, Brunelle F, Robert JJ, Nihoul-Fékété $C$, Saudubray JM, Junien C (1997) Somatic deletion of the imprinted $11 \mathrm{p} 15$ region in sporadic persistent hyperinsulinemic hypoglycemia of infancy is specific of focal adenomatous hyperplasia and endorses partial pancreatectomy. J Clin Invest 100:802-807

11. Verkarre V, Fournet J-C, de Lonlay P, Gross-Morand M-S, Martine Devillers J, Rahier FB, Robert J-J, Nihoul-Fékété C, Saudubray J-M, Junien C (1998) Paternal mutation of the sulfonylurea receptor (SUR1) gene and maternal loss of $11 \mathrm{p} 15 \mathrm{im}$ printed genes lead to persistent hyperinsulinism in focal adenomatous hyperplasia. J Clin Invest 102:1286-1291

12. Braune $K$, Wäldchen $M$, Raile $K$, Hahn $S$, Ubben $T$ Römer S, Hoeber D, Reibel NJ, Launspach M, Blankenstein O, Bührer C (2020) Open-source technology for real-time continuous glucose monitoring in the neonatal intensive care unit: case study in a neonate with transient congenital hyperinsulinism. J Med Internet Res 22:e21770

13. Palladino AA, Bennett MJ, Stanley CA (2008) Hyperinsulinism in infancy and childhood: when an insulin level is not always enough. Clin Chem 54:256-263

14. States LJ, Saade-Lemus S, De Leon DD(2020) 18 F-L 3,4-Dihydroxyphenylalanine PET/computed tomography in the management of congenital hyperinsulinism. PETClin 15:349-359

15. Prasad V, Sainz-Esteban A, Arsenic R, Plöckinger U, Denecke T, Pape UF, Pascher A, Kühnen P, Pavel M, Blankenstein O. (2016) Role of (68)Ga somatostatin receptor $\mathrm{PET} / \mathrm{CT}$ in the detection of endogenous hyperinsulinaemic focus: an explorative study. Eur J Nucl Med Mol Imaging 43:1593-1600

16. Boss $M$ et al (2021) 68Ga-NODAGA-exendin-4 PET improves the detection of focal congenital hyperinsulinism. J Nucl Med. https://doi.org/10. 2967/jnumed.121.262327

17. Meissner T, Wendel U, Burgard P, Schaetzle $S$ Mayatepek E (2003) Long-term follow-up of 114 patients with congenital hyperinsulinism. Eur JEndocrinol 149:43-51

18. Mercimek-Mahmutoglu S, Rami B, Feucht $M$, Herle $M$, Rittinger 0 , Stoeckler-Ipsiroglu $S$, Schober E. (2008) Long-term follow-up of patients with congenital hyperinsulinism in Austria. JPediatr Endocrinol Metab 21:523-532

19. Mohnike K, Blankenstein O, Christesen HT, De Lonlay J, Hussain K, Koopmans KP, Minn H, Mohnike W, Mutair A, Otonkoski T, Rahier J, Ribeiro M, Schoenle E, Fékété C. (2006) Proposal for a standardized protocol for 18F-DOPA-PET (PET/CT) in congenital hyperinsulinism. Horm Res $66: 40-42$

Hinweis des Verlags. Der Verlag bleibt in Hinblick auf geografische Zuordnungen und Gebietsbezeichnungen in veröffentlichten Karten und Institutsadressen neutral. 